\title{
Intermédialités
}

Histoire et théorie des arts, des lettres et des techniques

Intermediality

History and Theory of the Arts, Literature and Technologies

\section{L'art comme remise en scène de la vie quotidienne : traduire le social en esthétique}

\section{Camille Bui}

Numéro 27, printemps 2016

traduire

translating

URI : https://id.erudit.org/iderudit/1039810ar

DOI : https://doi.org/10.7202/1039810ar

Aller au sommaire du numéro

Éditeur(s)

Revue intermédialités (Presses de l’Université de Montréal)

ISSN

1920-3136 (numérique)

Découvrir la revue

Citer cet article

Bui, C. (2016). L'art comme remise en scène de la vie quotidienne : traduire le social en esthétique. Intermédialités / Intermediality, (27).

https://doi.org/10.7202/1039810ar
Résumé de l'article

Cet article explore, à travers le paradigme de la traduction, la mise en scène des interactions sociales dans Playtime de Jacques Tati (1967), Kontakthof de Pina Bausch (1978-2000-2008) et Reconstitutions d'Édouard Levé (1998-2003). Chacune de ces oeuvres témoigne du désir de traduire le social en esthétique : il s'agit de dire l'ordinaire des jeux de distance et de proximité entre les corps, par le cinéma, la danse ou la photographie. Ce geste de traduction emprunte à la logique de la re-présentation qui réifie le social, mais il ne peut s’y réduire. Car la traduction vise aussi à reconduire, sur la scène de l'art, la dynamique du lien en tant qu'adresse faite à l'autre, au risque de son échec. Penser le geste artistique comme traduction permet d'être sensible au devenir chorégraphique des situations sociales, autant qu’à la socialité qui anime des oeuvres qui nous émeuvent ou nous indiffèrent. 


\title{
L'art comme remise en scène de la vie quotidienne : traduire le social en esthétique
}

\author{
Camille Bul
}

\section{MANIÈRES DE TRADUIRE}

n 1998, dans son œuvre vidéographique intitulée The Pitch, le Canadien Mark Lewis se mettait en scène au milieu de l'agitation d'un hall de gare, lisant un texte qui se portait à la défense des figurants, ces corps silencieux qui traversent les films sans se faire remarquer (voir la figure I). La vidéo s'ouvre sur le visage de l'artiste filmé en gros plan. Puis, la caméra dézoome peu à peu, jusqu’à ce que son corps ne soit plus qu'une silhouette mêlée à la foule des voyageurs avec leur valise, marchant d'un pas lent ou pressé, s'arrêtant devant le tableau d'affichage des départs. À mesure que l'on quitte l'échelle des plans individualisante, l'attention se déplace sur l'aléatoire ballet des passants. Cette œuvre pointe le statut effacé qu'occupent les « extras » dans l'industrie cinématographique et met en lumière l'échec du cinéma dominant à rendre sensible la trame de l'ordinaire. D'autres artistes ont opposé à la mécanique du récit classique et à la hiérarchie des corps qu'elle semble dicter une attention à ce qu'Erving Goffman, dans son discours sociologique, appelait «l'ordre de l'interaction », soit le fonctionnement des situations d'interaction quotidiennes. En particulier, le cinéma dit moderne ainsi que la danse et la photographie contemporaines ont souvent fait écho à ce projet, proche des interrogations de Perec sur «l'infra-ordinaire » : Ce qui se passe chaque jour et qui revient chaque jour, le banal, le quotidien, l'évident, le commun, l'ordinaire, le bruit de fond, l'habituel, comment en rendre compte, comment l'interroger, comment le décrireı ? ». Et, pourrait-on ajouter, comment le traduire?

\footnotetext{
I. Georges Perec, L'infra-ordinaire, Paris, Éditions du Seuil, 1989, p. 9.
} 


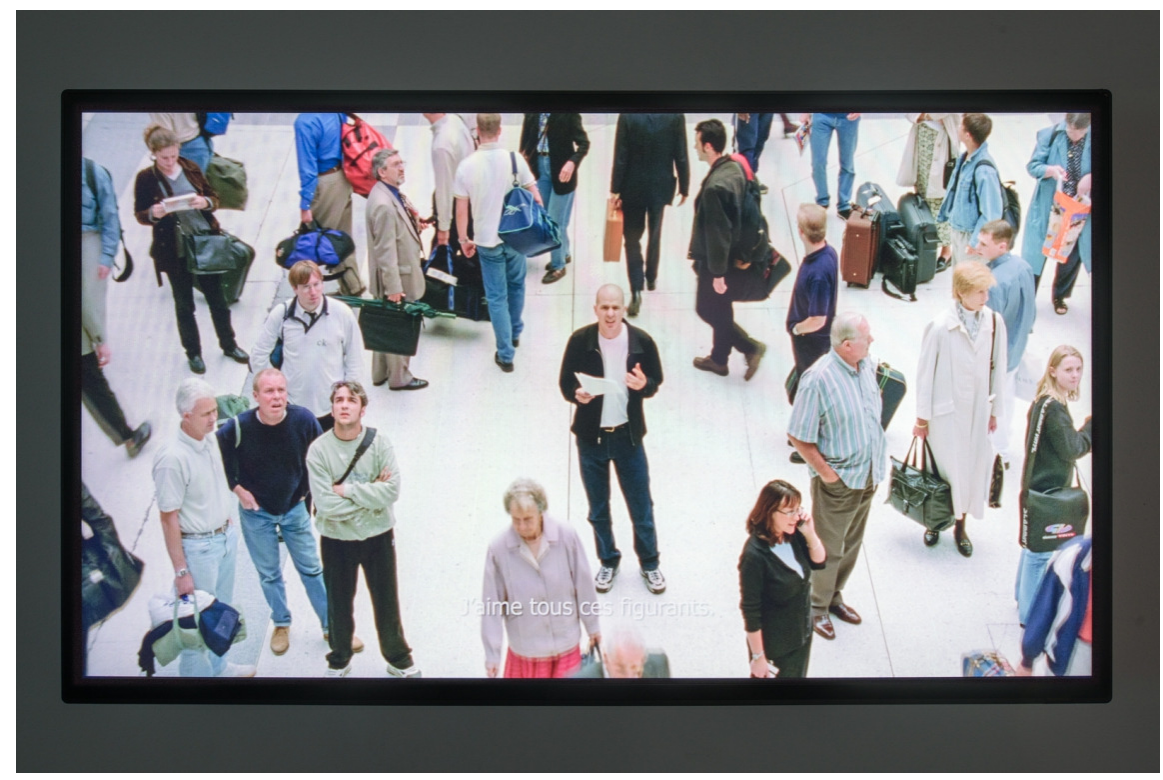

Fig. I. Mark Lewis, The Pitch, 1998, vue de l'exposition « Mark Lewis - Above and below » du 5 février au 17 mai 20I5, LE BAL, Paris.

\section{LE BAL @ Martin Argyroglo}

J'aimerais, dans ce texte, interroger en tant qu'opération de traduction le rapport entre la socialité ordinaire - faite des liens faibles autant que des amours ou des déprises de n'importe qui - et ses formes esthétisées dans le champ artistique. Je propose pour cela de mettre en relation le film Playtime (1967) de Jacques Tati avec la pièce chorégraphique Kontakthof (1978-2000-2008) de Pina Bausch et les séries photographiques intitulées Reconstitutions d'Édouard Levé (1998-2004). Ces créateurs s'inscrivent dans des filiations distinctes : cinéma burlesque chez Tati; héritage des chorégraphes Rudolf von Laban et Kurt Jooss, mais aussi du théâtre de Brecht et d'Artaud chez Bausch; modernité picturale et art conceptuel chez Levé. Néanmoins, il existe une correspondance dans la manière dont tous trois envisagent les interactions humaines en tant que phénomènes sensibles, rituels composés de gestes, de déplacements, de regards et de postures, avant que de recourir à des mots.

Réfléchir à un spectacle de tanztheater, à un long-métrage de fiction et à une série photographique à l'aune du concept de traduction ne va pas de soi, s'agissant d'œuvres créées dans des médiums non strictement linguistiques qui, de surcroît, mettent ici en scène un matériau quotidien en majeure partie non verbal. Toutefois, si la danse, le cinéma et la photographie ne sont pas à proprement parler des langues et possèdent chacun une puissance dénotative différente, ils ont en commun avec l'ordre 
de l'interaction de constituer des pratiques codifiées dont la structure - en particulier lorsqu'elle se déploie dans le temps — est souvent comparée à celle d'un langage ${ }^{2}$. Mais, plus que d'interroger la nature - métaphorique ou non - de ces «langues » artistiques, ce que je souhaite, c'est de me saisir ici de la notion de traduction dans une perspective heuristique. Il s'agit d'explorer en quoi le paradigme de la traduction peut nous aider à penser ce qui, dans le lien entre le social et l'esthétique, ne peut se réduire à une opération simple de re-présentation, entendue comme passage d'un réel vécu à l'image de ce vécu. En envisageant chaque mise en scène comme une manière de traduire que l'analyse se donne pour tâche de qualifier, de définir, j'aimerais mettre au jour la complexité de la relation qui se tisse entre l'expérience sociale ordinaire et l'expérience esthétique.

J'envisage donc ici représentation et traduction comme deux pôles d'un continuum parcouru par une tension qui peut être posée ainsi : dans quelle mesure la « mise en scène de la vie quotidienne » dans un espace qui lui est a priori hétérogène constitue-t-elle un processus violent de réification de la socialité ? Peut-elle se faire au contraire le rebond d'une expérience dans une autre et ainsi reconduire, sur la scène de l'art, la dynamique vivante de l'interaction et son horizon communautaire ?

Nous explorerons dans un premier temps le devenir chorégraphique commun des images de la socialité proposées par Bausch, Tati et Levé. Puis, en faisant un pas du côté de la genèse des œuvres, nous nous pencherons sur un paradoxe : le désir de mettre en scène de façon soigneusement contrôlée des interactions pourtant définies par leur imprévisibilité et leur vulnérabilité. Enfin, en interrogeant la forme de l'adresse faite au spectateur, nous verrons la façon dont les auteurs s'attachent en réalité à déplacer dans l'expérience esthétique elle-même la force des liens vécus, autant que le risque de leur ratage.

\section{CADRER LA MISE EN SCÈNE SOCIALE}

Dès la sortie des Vacances de M. Hulot, André Bazin soulignait le contraste entre le personnage inventé par Jacques Tati et ses ancêtres burlesques des années 1920

2. Autour du cinéma comme langage, on pourra se référer au texte classique de Christian Metz. Christian Metz, «Le cinéma: langue ou langage ? », Communications, 1964, vol. 4, n I, p. 52-90. Sur cette question, à propos de la danse, voir Henrietta Bannerman, «Is Dance a Language? Movement, Meaning and Communication », Dance Research, vol. 32, n I, p. 65-80; à propos de la photographie, voir Victor Burgin, «Looking at Photographs », dans Victor Burgin (dir.), Thinking Photography, Macmillan Education UK, 1982, p. I42-I53. 
et 1930, héritiers de la commedia dell'arte, tout en présence virtuose, tels Charlot et les Marx Brothers. Pour Bazin, l’originalité du personnage de Tati « réside dans son inachèvement »: «Le propre de M. Hulot semble être de ne pas oser exister tout à fait. Il est une velléité ambulante, une discrétion d'être. Il élève la timidité à la hauteur d'un principe ontologique 3 . »

C'est ainsi que, de film en film, M. Hulot est amené à disparaître par intermittence pour laisser le commun des mortels investir le champ. En 1967, dans Playtime, il n'est presque plus qu'un point d'entrée ou de sortie dans des situations sociales dont il va jusqu'à s'absenter. Dans le va-et-vient du hall de l'aéroport, parmi l'agitation du salon industriel, à la soirée dansante au restaurant Royal Garden, Hulot ne fait souvent que passer ou attendre parmi les citadins et les touristes affairés. Ce sont les rencontres fugitives, les rassemblements, les parcours et détours quotidiens, les frôlements, les malentendus et les heureux hasards - toutes choses généralement reléguées à l'arrière-plan du drame - qui forment le cour de la vie urbaine dans Playtime, tandis que Hulot, « devenu le badaud absolu ${ }^{4} »$, subit, comme « tout le monde $\gg$, le cours des événements 5 .

Transposée à la danse classique, la question de la distribution des corps sur la scène se pose en des termes qui font écho à ceux du cinéma. C’est ce que le chorégraphe Jérôme Bel soulignait dans sa pièce Véronique Doisneau (2004), du nom de la danseuse du corps de ballet de l'Opéra de Paris qui y apparaît. Seule sur scène, Doisneau est invitée à témoigner de son parcours professionnel. En contrepoint, elle exécute des séquences chorégraphiques d'œuvres classiques, telles Le lac des cygnes, chorégraphié par Rudolf Noureev, ou Giselle, par Jean Coralli et Jules Perrot. Mais, n'étant pas une danseuse étoile, la plus grande partie de sa performance consiste à réaliser de brefs mouvements ou à rester longtemps immobile à l'arrière-plan, dans des poses gracieuses, en feignant de ne faire aucun effort. Ce changement de focalisation, à la fois comique et pathétique, rend visible les rapports de pouvoir sous-jacents à la danse classique, avec lesquels de nombreux chorégraphes du $20^{\mathrm{e}}$ siècle ont cherché à rompre : parmi eux, Pina Bausch, formée à Essen dans les années 1950 à la Folkwang Hochschule, école d'art pluridisciplinaire alors dirigée par Kurt Jooss, puis à New York au tournant des

3. André Bazin, « M. Hulot et le temps » [1953], dans Qu'est-ce que le cinéma? Paris, Les Éditions du Cerf, I985, p. 43.

4. Michel Chion, Jacques Tati, Paris, Cahiers du Cinéma, coll. « Auteurs », 1987, p. 64.

5. Jacques Tati, «Entretien avec Jacques Tati, par André Bazin et François Truffaut», Cabiers du cinéma ${ }^{\circ} 83$, mai 1958 , p. 4. 
années 1960 avec José Limón, Antony Tudor ou Paul Taylor. Selon les termes de Brigitte Gauthier, la chorégraphe allemande

hérite [d'un] courant de pensée qui détache le danseur de la soumission à la chorégraphie. Il n'est plus fondu dans la masse, simple rouage d'une mécanique classique qui ne laisse affleurer sur le devant de la scène que le soliste. Son œuvre met certes en valeur des solos [...] [, $\mathrm{m}$ ] ais [ceux-ci] ne se détachent pas sur fond de séquences dansées par des individus non distincts comme traditionnellement dans la danse classique ${ }^{6}$.

À la liberté des corps chez Tati vis-à-vis de la ligne d'action narrative répond celle des danseurs de Pina Bausch par rapport à la chorégraphie. Kontakthof, pièce de théâtre dansé autour d'une question majeure de son œuvre, « Vouloir être aimé - tout ce que nous faisons pour que quelqu'un nous aime », met en scène des interactions entre hommes et femmes, variations gestuelles explorant des situations de séduction, de joie, d'intimité amoureuse ou sexuelle, de pouvoir et de violence, de solitude ou de désespoir. C'est souvent l'ensemble des danseurs qui occupe la scène. En tenue de soirée, ils dansent en groupe, comme lors de cette séquence où se font face, en deux rangs, les hommes, assis sur des chaises à gauche de la scène, et les femmes, debout contre le mur opposé. Les premiers s'approchent des secondes en projetant compulsivement leurs bras en avant pour les saisir, tandis qu'elles se dérobent à leur emprise. Face-à-face, cercle, ligne tournée vers le public: ces motifs collectifs reviennent, mais sont souvent rompus. Seuls ou en couples, les danseurs s'avancent parfois tour à tour sur le devant de la scène, comme s'ils effectuaient pour l'assistance un petit numéro, avant de revenir en fond de scène et de réintégrer le groupe. Ou bien, un seul danseur se trouve isolé du groupe, ou chaque paire s'adonne à un mouvement ou à une rencontre dans une bulle séparée. Fragmentant le temps et l'espace, ces actions simultanées ou successives invitent le spectateur à changer sans cesse de point de focalisation (voir les figures 2-4).

6. Brigitte Gauthier, Le langage chorégraphique de Pina Bausch, Paris, L'Arche Éditeur, 2008 , p. 37 . 

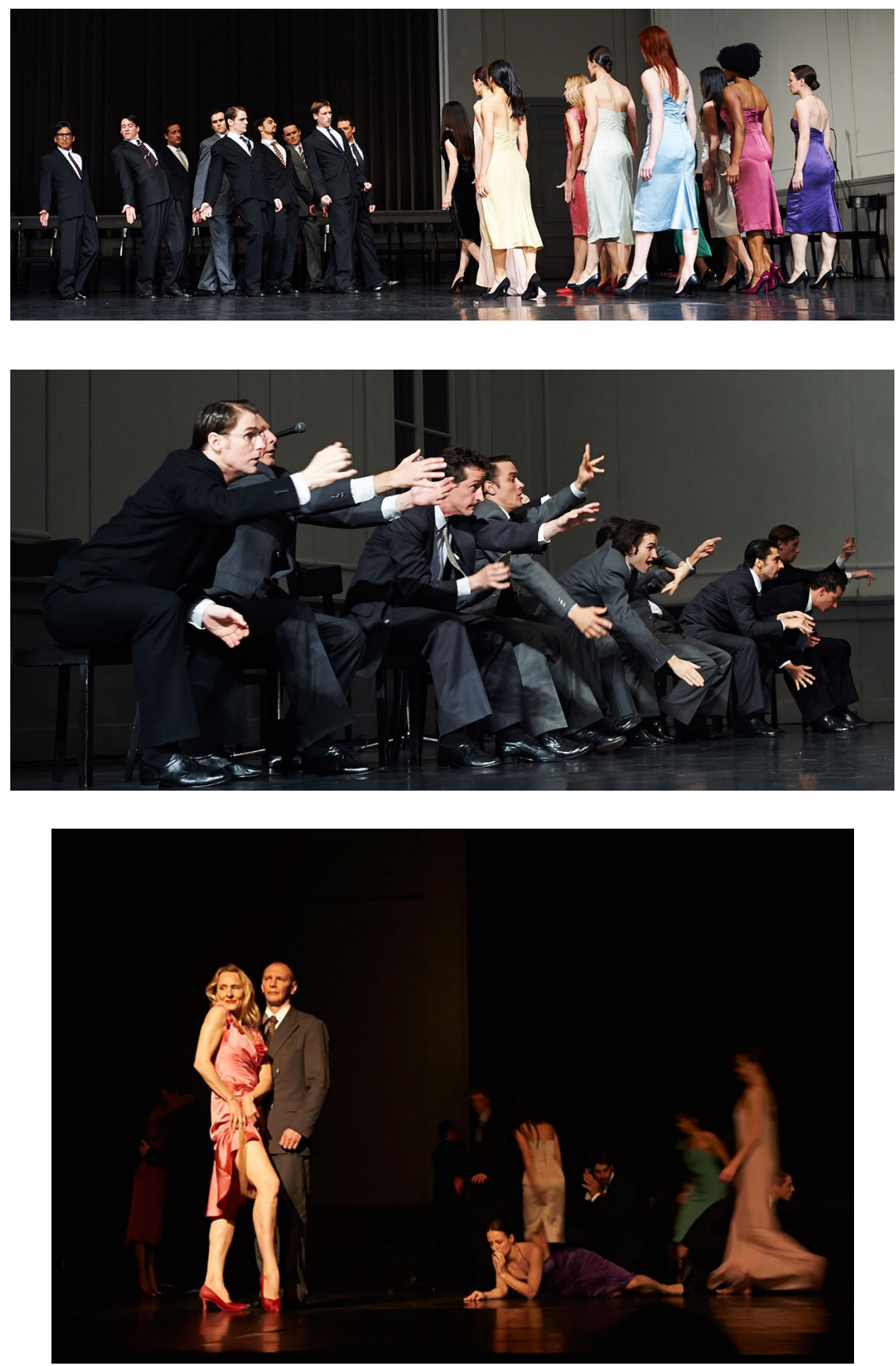

Fig. 2-4. Pina Bausch/Tanztheater Wuppertal, Kontakthof, 1978-2000-2008, Luminato Festival, Toronto, 11-14 juin 2014 .

(C) David Leyes 
D’une autre façon, les Reconstitutions d'Édouard Levé se présentent comme des images de groupe. Selon les mots de l'artiste, l'enjeu de ses séries photographiques est de donner à observer « les personnes, la façon dont elles communiquent entre elles, ou sont prises dans une action ${ }^{7} »$. Chaque cliché est le résultat d'une « digestion » mentale par l'artiste de dizaines d'images appartenant à un même genre iconographique. Sur fond neutre, clair ou sombre, en tenue de ville, des modèles au corps figé et au visage inexpressif adoptent les poses caractéristiques d'hommes politiques, d'acteurs pornographiques ou de joueurs de rugby (voir la figure 5 ).

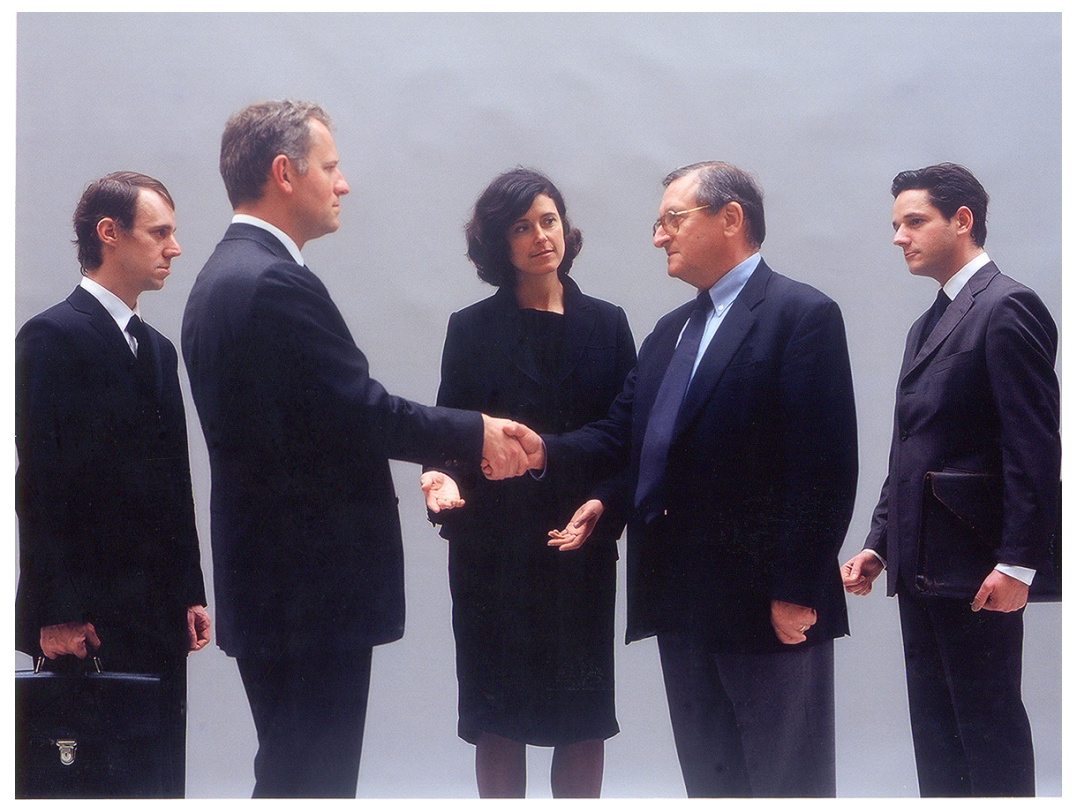

Fig. 5. Édouard Levé, série Reconstitutions - Actualités, La Rencontre, 200I, photographie couleur, 68 x $100 \mathrm{~cm}$, édition à 5 exemplaires. Reproduit avec l'aimable autorisation de la galerie Loevenbruck, Paris.

En reproduisant de manière décontextualisée les apparences d'événements qui avaient été mis en scène pour ou par un appareil photo, Levé propose une traduction du social au second degré. Il s'agit d'extraire de cette imagerie sa part d'ordinaire pour, par le stéréotype, interroger les rapports de pouvoir qui régissent la disposition des corps dans le cadre. En supprimant tout indice circonstanciel et en retournant la logique de «l'instant décisif » en un temps générique suspendu, Levé concentre notre attention

7. Édouard Levé, « Interview d'Édouard Levé par lui-même », dans Reconstitutions, Paris, Phileas Fogg Éditions, 2003, p. 84. 
non plus sur tel personnage politique, érotique ou sportif, ni sur un événement brûlant de l'actualité, mais sur la chorégraphie rituelle de corps abstraits, qui valent pour leur caractère symbolique. Notre regard embrasse la composition d'ensemble ou bien parcourt le réseau des relations visuelles et gestuelles qui, tels des fils invisibles, lient les modèles les uns aux autres - et ce, sans que nous butions véritablement sur un punctum.

Chez Tati également, la dissolution du personnage principal va de pair avec la circulation libre du regard du spectateur dans l'espace «scénique », d'un corps à l'autre. Michel Chion remarque en effet que, chez le cinéaste comique, « l'ignorance [délibérée] des règles habituelles du découpage », combinée à « l'emploi systématique du plan général, interdit la classique identification au personnage ${ }^{8} »$. Ce $\ll$ refus du gros plan » associé à la très grande netteté rendue possible par la pellicule $70 \mathrm{~mm}$ de Playtime permet à l'attention de « voyager entre les personnages » qui emplissent le cadre $^{9}$. La séquence centrale de la soirée au Royal Garden voit ainsi le champ cinématographique se transformer littéralement en espace chorégraphique où différents couples évoluent simultanément. Le choix de ne pas trancher dans la chair des situations par des plans de coupe ou des champs-contrechamps inverse la logique habituelle de l'énonciation filmique qui impose son rythme et son cadre aux corps des acteurs $^{\mathrm{IO}}$. C'est ici le corps collectif qui, par ses mouvements, anime la rigidité du cadre social et cinématographique. Comme chez Bausch, les co-présences sur la « scène » donnent naissance à l'image d'une communauté composée d'individus distincts, dont les actions conjointes remodèlent sans cesse l'espace.

Ces traductions de situations sociales en situations esthétiques ne sont pas sans rapport avec la traduction sociologique qu'en propose Erving Goffman tout au long de son œuvre, consacrée au « domaine du face-à-face [...], dont la méthode d'analyse préférée est la micro-analyse ${ }^{\mathrm{II}} \gg$. En déjouant la hiérarchie classique entre personnages, danseurs ou sujets photographiés, les trois œuvres étudiées adoptent le point de vue de la situation sociale plutôt que ceux d'individus isolés. Leurs cadrages donnent à voir

8. Chion, 1987, p. 5 I.

9. Ibid., p. 52.

Io. Vincent Amiel, Le corps au cinéma. Keaton, Bresson, Cassavetes, Paris, Presses Universitaires de France, 1998, p. III.

II. Erving Goffman, «L'ordre de l'interaction », Les moments et leurs hommes, Paris, Les Éditions de Minuit, 1988, p. I9I. Pour une analyse détaillée des liens entre l'œuvre de Goffman et le cinéma, voir Barbara Olszewska, « La sociologie cinématographiée d'Erving Goffman », dans Daniel Cefaï et Laurent Perreau (dir.), Erving Goffman et l'ordre de l'interaction, AmiensParis, CURAPP-CESS/CEMS-IMM, 2012, p. 299-322. 
l'engagement et la responsabilité conjointe des participants dans l'interaction ainsi que la définition réciproque de leurs rôles au cours de celle-ci. Mais, à la différence de la sociologie, la photographie, la danse et le cinéma ont en commun de traduire ces situations par la remise en scène de corps réels qui ne sont jamais tout à fait des signes, et ce, même si leurs présences revêtent des consistances différentes. Chez Bausch, les corps sont sensuels, pesants, désirants, alors que chez Tati, les présences physiques sont stylisées en silhouettes graphiques ${ }^{12}$ et que chez Levé, les êtres vivants se trouvent comme changés en statues. À l'abstraction de motifs génériques qu'ils partagent avec la démarche microsociologique, les artistes allient une singulière ré-incarnation de leur pensée du social.

\section{REJOUER LA DISTANCE ENTRE LES CORPS}

Ce double mouvement est particulièrement apparent si l'on se penche sur le traitement que Tati, Bausch et Levé font de la communication verbale. En effet, leurs traductions du social minimisent la dimension référentielle des énoncés au sein des situations mises en scène pour insister sur leur matérialité ou leur fonction poétique.

De nombreux critiques ont souligné l'importance du travail sonore chez Tati, tout en remarquant sa proximité artistique avec le cinéma muet. Dans Playtime, les dialogues sont souvent traités comme des bruits. Par exemple, lorsque Hulot - personnage désormais sans voix - entre dans l'immeuble pour rencontrer le mystérieux Giffard, la voix du gardien se fait entendre par bribes, presque par onomatopées (« Ouais, ouais, ouais, ouais... Bon, alors, hum, hum... Là... Alors, Monsieur, si vous voulez bien, je... m’occupe de vous, j’en ai pour deux minutes »). La voix est recouverte par un bourdonnement électrique continu qui la rend difficilement compréhensible, et par-dessus lequel résonnent le son des pas sur le marbre, le bruit du fauteuil sur lequel Hulot s'assoit, puis les bips électroniques de l'énorme console qui permet à l'employé de communiquer avec les étages supérieurs. Nous ne saisissons que le caractère général de la situation : Hulot attend un rendez-vous. Comme dans l'ensemble de Playtime, les « dialogues » sont essentiellement réduits à leur dimension phatique.

Le rôle mineur attribué à la communication verbale, ou du moins à sa fonction référentielle, participe à la chorégraphisation du social, traduit comme une oscillation des distances entre des corps sonores qui ne coïncident jamais tout à fait. À la difficulté des interactions en face à face s'oppose chez Tati le fonctionnement fluide de la

I2. Voir Chion, 1987, p. 77. 
communication moderne et capitaliste. Chion remarque que, dans Playtime, « les seules vraies voix qui portent, et dont le message pourrait venir », sont les « voix amplifiées et relayées sorties de haut-parleurs : ces voix ignorent la distance et, trouant le décor de part en part, peuvent naître en n'importe quel point de l'espace, qu'elles irréalisent ${ }^{13}$ ». Alors qu'ailleurs, « la matérialité du message [paraît] importer plus que son contenu $\mathrm{u}^{\mathrm{I} 4} \gg$, comme en témoigne le traitement de la différence linguistique dans Playtime qui ne fait l'objet d'aucune traduction entre les personnages. La mise en forme du social en esthétique insiste donc ici sur l'inadéquation fondamentale des êtres à eux-mêmes et aux autres, mais aussi à leur environnement technique où, paradoxalement, «tout communique », pour reprendre la célèbre réplique de Mon oncle (1958). Il n'y a nulle fusion organique chez Tati, mais un plaisir poli à être la victime de quiproquos ou de malentendus.

Chion qualifie avec finesse les scénarios sociaux chez Tati d'« histoires d'éviter ${ }^{15} \gg:$ le lien entre les corps se tisse à distance, presque secrètement, comme entre Hulot et la jeune Américaine, touriste de passage, qui se croisent de loin, par « événements et accidents qui se transmettent par contamination ${ }^{16}$ ». Lorsque les deux personnages discrets se retrouvent ensemble sur la piste de danse du Royal Garden, c'est qu'ils se sont chacun laissés entraîner dans des mouvements de groupe. Et durant cette soirée où, au rythme de la bossa-nova puis du free jazz, les corps bourgeois disciplinés se laissent aller à subvertir les conventions sociales, rien de charnel ne circule véritablement entre eux, et le « cadre » n'explose jamais tout à fait.

Chez Tati, le social apparaît comme un ordre des séparations - des genres, des classes, des cultures et des individus - qui rend possible un rapprochement éphémère : autrement dit, «c'est une fois le tri fait (...) que peut s'ébranler cette centrifugeuse qu'est la machine du film ${ }^{17} \gg$. L'interaction prend chez lui la forme d'un jeu de distances à la fois tendre et mélancolique, d'une jouissance contenue, faisant écho à la vision burlesque du monde industriel, soit « un art de vivre dans un monde toujours sur le point de se déborder lui-même absurdement, scandaleusement ${ }^{18} \gg$, selon les termes de Jean-Philippe Tessé.

13. Chion, 1987, p. I02.

I4. Ibid., p. I05.

I5. Ibid., p. 69.

16. Ibid., p. 7I.

17. Ibid., p. 68.

I8. Jean-Philippe Tessé, Le burlesque, Paris, Cahiers du Cinéma, coll. « Les Petits Cahiers », 2007 , p. 65. 
On retrouve chez Bausch, de manière plus crue, incarnée, pulsionnelle, ce désir de communiquer en deçà ou au-delà d'un contenu référentiel toujours fragmenté, déstructuré, parfois absurde. Dans ce théâtre dansé, avant d'être locutoires, les paroles sont des actes engageant tout le corps de celle ou celui qui les prononce. Elles traduisent une modalité d'être: désir soudain d'exprimer un désir ou une souffrance, d'être regardé, d'avouer quelque chose. Dans une séquence de Kontakthof, tous les danseurs sont alignés sur des chaises et s'adressent au public en remuant les lèvres. L'un d'entre eux leur tend tour à tour un micro, faisant résonner dans la salle quelques phrases de chaque témoignage, avant de passer au suivant. Du fait de leur interruption et de leur succession, ces fragments d'histoires personnelles s'évanouissent rapidement, et l'attention se porte essentiellement sur les attitudes des corps. Ce qui se donne à voir ici, c'est d'abord une impulsion vers l'autre, une adresse, indépendante de la réception d'un message. Le micro, objet instaurant une asymétrie dans la communication entre celui qui parle et celui qui écoute, est souvent mobilisé dans Kontakthof comme incarnation de cette demande d'amour et de reconnaissance.

$\mathrm{La}$ (non-)circulation de la parole chez Bausch s'intègre donc plus globalement aux jeux de proximité et de distance qui projettent sans cesse les corps des femmes et des hommes les uns vers les autres, les entraînent ensemble dans des petits pas de danse, des rondes mécaniques ou des courses effrénées, font que les regards et les caresses tendres se convertissent en brimades minuscules ou en agressions sadiques. Chez Tati, la distance entre les corps apparaissait comme le donné d'une solitude moderne pardessus laquelle se lier tout en laissant « de l'air » entre soi et les autres. Chez Bausch, les corps s'acharnent sans relâche à franchir cette distance douloureuse, à la combler, quitte à danser avec un absent. Dans Kontakthof, littéralement « cour de contact », la douceur du rapprochement risque toujours de se retourner en brutalité de la collision et des rapports de pouvoir (voir les figures 6 et 7 ).

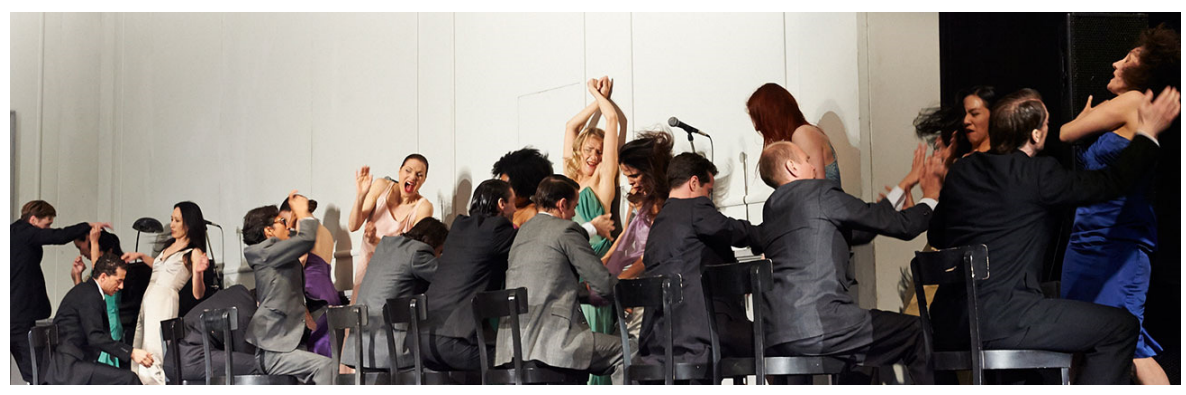

Fig. 6. Pina Bausch/Tanztheater Wuppertal, Kontakthof, 1978-2000-2008. Luminato Festival, Toronto, II-I4 juin 2014.

(c) David Leyes 


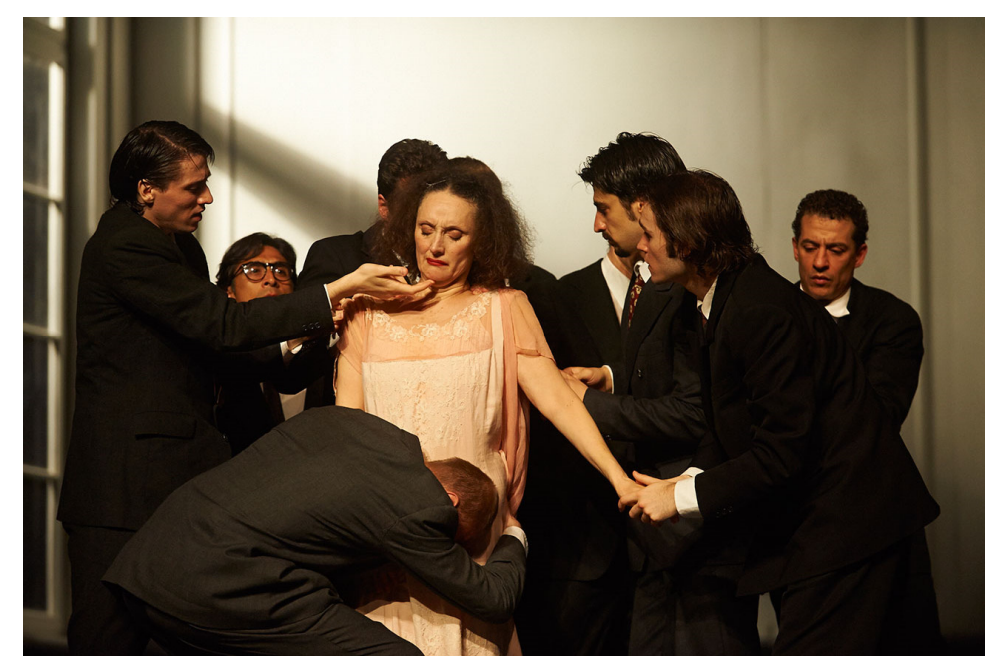

Fig. 7. Pina Bausch/Tanztheater Wuppertal, Kontakthof, 1978-2000-2008. Luminato Festival, Toronto, $\mathrm{II}-\mathrm{I} 4$ juin $2 \mathrm{OI} 4$.

(c) David Leves

Ainsi, si chez Tati tout est en retenue, chez Bausch, la difficulté de la rencontre prend forme dans des corps expressifs, parfois jusqu'à l'hystérie. Par rapport à une danse qui tente de réchauffer les solitudes, la photographie semble agir comme une machine de refroidissement. Chez Levé, la distance inhérente aux rapports humains devient glaciation mécanique des corps en présence, ce qui, là encore, minimise la dimension discursive du social.

Par nature, le médium photographique ne joue pas sur le même terrain que les arts du spectacle à l'endroit du corps parlant. Pour Barthes, la photographie se caractérise précisément par le « rien à dire » de son « évidence muette ${ }^{19}$ ». Toutefois, si le médium est littéralement silencieux, ses usages sociaux le voient souvent accompagné d'indications textuelles visant à le tirer de son mutisme. Or, dans les Reconstitutions, les remises en scène de situations archétypales photographiées par la presse ne sont plus accompagnées que du titre générique de la série. L'absence de légende ou de texte d'actualité, qui en « ancrerait le sen $\mathrm{s}^{20}$ » en orientant le spectateur dans le labyrinthe des interprétations possibles, redouble la décontextualisation

19. Voir Roland Barthes, La chambre claire, Paris, Cahiers du Cinéma, Gallimard, Le Seuil, 1980.

20. Voir Roland Barthes, « Rhétorique de l'image », Communication, vol. I, n 4 , I964, p. 40-5I. 
qu'opérait déjà la mise en scène plastique soustractive. Le gommage des messages médiatiques qui brouillent la visibilité des codes sociaux fait apparaittre la violence et l'absurdité de ces derniers.

Cela s'incarne en particulier dans les séries « Actualités » et « Pornographie ». En effet, dans les événements importants de la vie publique, ce sont des hommes blancs et âgés qui occupent les positions de pouvoir et le centre de l'image, tandis que les femmes sont soit absentes, soit en minorité, reléguées au second plan. Cette analyse iconographique semble aller en gradation : dans « Pornographie », les rapports de pouvoir entre genres apparaissent cette fois encore plus crûment, les femmes étant globalement soumises aux désirs des hommes. Le figement de la composition donne l'impression d'un ordre social immuable qui emprisonne les individus. Dans «Quotidien », le regard semble s'affranchir de tout discours explicite pour donner lieu à des tableaux étranges, plus proches du flottement sémantique de la série initiale intitulée « Rêves ». Cette fois, le code n'est plus lisible, et s'y substitue le sentiment d'un drame sans objet. Les gestes deviennent mystérieux, et l'inexpressivité des visages accentue ce sentiment de violence latente entre des corps tournés les uns vers les autres, si proches et pourtant indifférents à la situation qui les rassemble (voir les figures 8-I2).

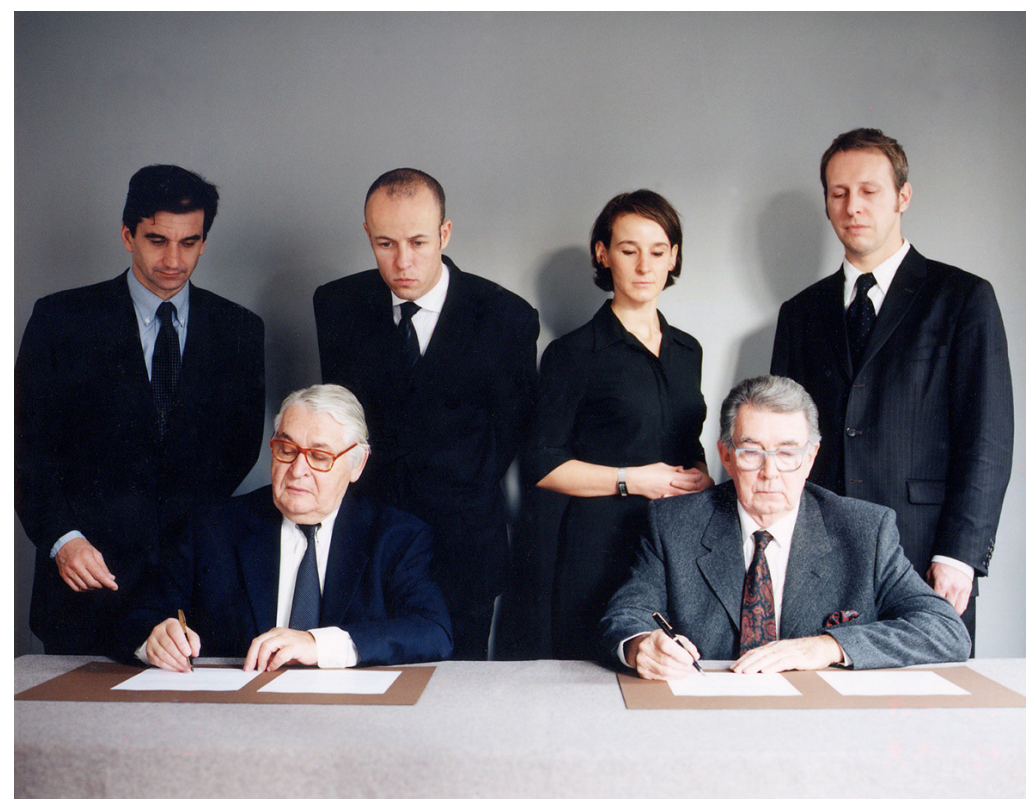

Fig. 8. Édouard Levé, série Reconstitutions - Actualités, L’Accord, 20oI, photographie couleur, $68 \times 100 \mathrm{~cm}$, édition à 5 exemplaires. Reproduit avec l'aimable autorisation de la galerie Loevenbruck, Paris. 

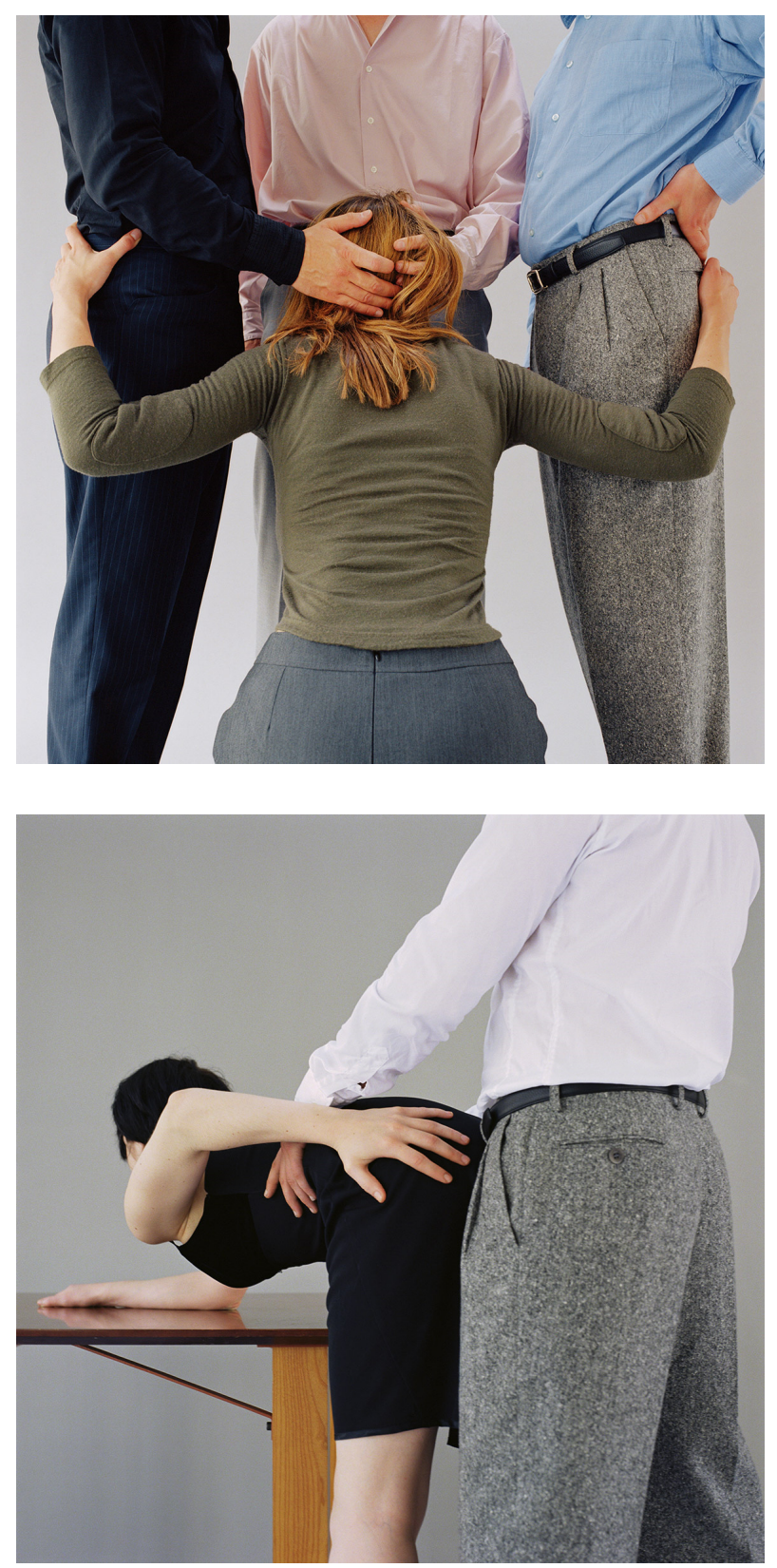

Fig. 9-Io. Édouard Levé, série Reconstitutions - Pornographie, Sans titre, 2002, photographie couleur, $70 \times 70 \mathrm{~cm}$, édition à 5 exemplaires. Reproduit avec l'aimable autorisation de la galerie Loevenbruck, Paris. 

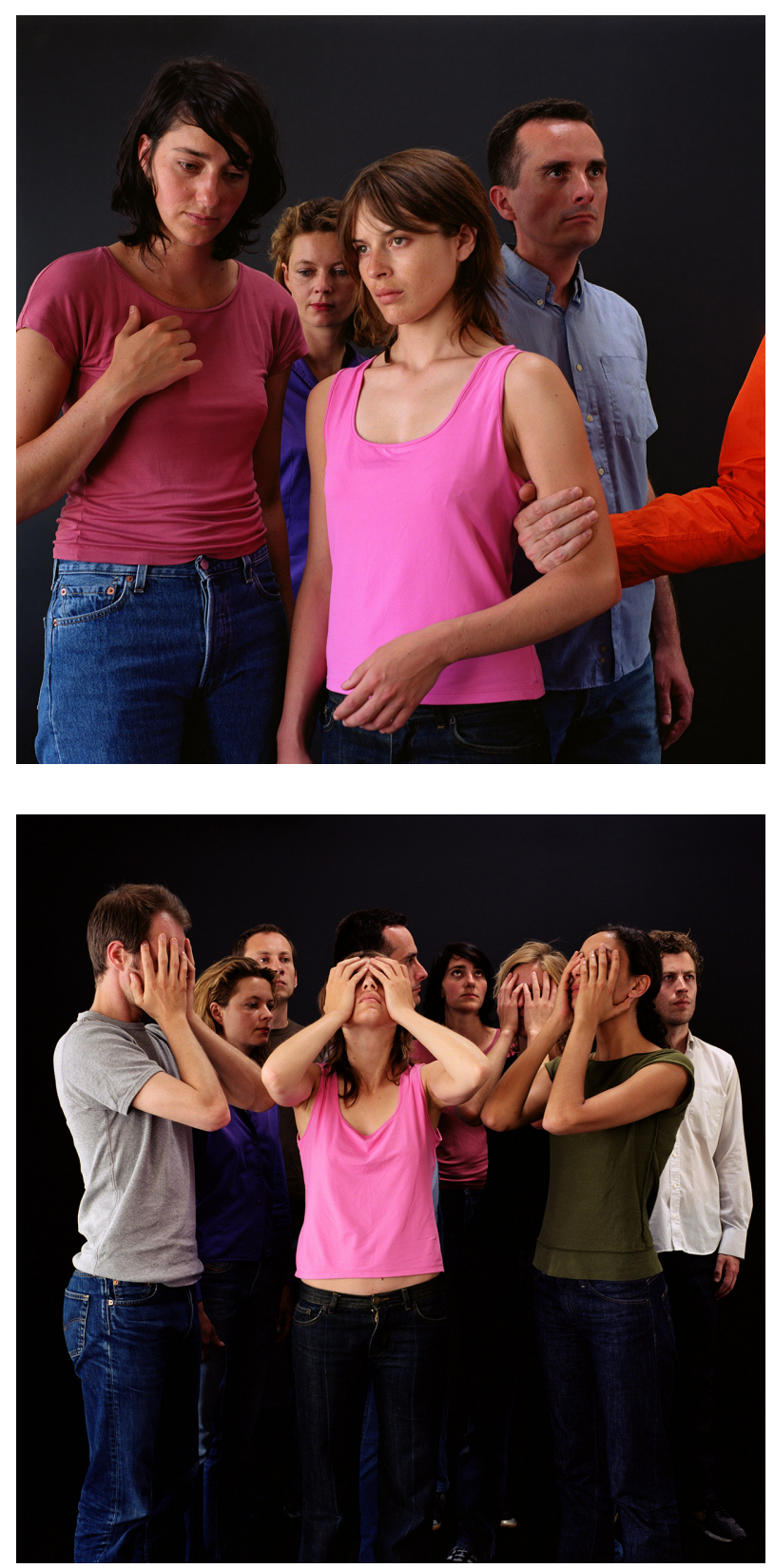

Fig. II-I2. Édouard Levé, série Reconstitutions - Quotidien, Sans titre, 2003, photographie couleur, 70 x $70 \mathrm{~cm}$, édition à 5 exemplaires.

Reproduit avec l'aimable autorisation de la galerie Loevenbruck, Paris. 


\section{LE DEVENIR CHORÉGRAPHIQUE DU SOCIAL} communauté dématérialisée, les trois œuvres qui nous intéressent sont d'abord sensibles à la part d'impossible, de brouillage, d'échec existant dans toute tentative d'interaction. Elles font apparaître que la communication est toujours performative et qu'elle est donc, comme tout acte humain, quelque chose «qui risque de $\operatorname{rater}^{2 \mathrm{II}}$ ». Mais cette mise en scène de la vulnérabilité advient, paradoxalement, en s'éloignant de la contingence et du risque qui fondent la socialité ordinaire. Car, à la différence de certaines pratiques basées sur l'improvisation — du cinéma documentaire à la fiction qui s'en inspire, de la dansecontact à la street photography —, l'attention de Tati, Bausch et Levé aux interactions sociales et à leur dimension infra-ordinaire s'incarne dans des mises en scène extrêmement ordonnées, qui penchent vers une forme réificatrice de représentation. personnage comique pour qu'il vous arrive une situation comique ${ }^{22}$. $\gg$ Le rire ne doit pas, selon lui, être le résultat d'un savoir-faire spécifique comme dans le cirque ou le music-hall, mais d'une situation qui se déroule malgré celui qui en est à la fois l'artisan et la victime, tel cet homme sérieux coinçant sa cravate dans la portière de sa voiture. Le travail du cinéaste commence par une sensibilité à ce comique ordinaire, celui dont n'importe qui peut être responsable ou spectateur. Le paradoxe de Tati consiste ainsi à remettre en scène par un geste artistique entièrement maîtrisé ce genre de situations ordinaires. En tant qu'acteur, son talent réside dans sa capacité à jouer activement la passivité, le corps pris dans les contingences quotidiennes : jouer la confusion de celui qui manque son ascenseur, la discrétion de celui qui se glisse, gêné, entre deux portes, l'étonnement de celui qui arrive malgré lui dans un dancing, l'incompréhension polie de la victime d'un quiproquo. Si la situation diégétique échappe à la volonté du personnage, la performance de l'acteur-cinéaste est, elle, parfaitement réglée. Les moindres petits gestes des acteurs sont minutieusement planifiés, comme en témoigne ce récit du tournage de Mon oncle:

Sur le plateau, je sais exactement ce que je vais demander aux acteurs, ce que je vais faire (...) je n'improvise pas. Je sais tout à l'avance. Le montage, aussi, je le fais à l'image. Je monte le film par cœur. Vraiment, je vous assure, dans ce film j'ai fait tout ce que j'ai voulu. Si on n'aime pas ça, c'est à moi seul qu'il faut s'en prendre ${ }^{23}$.

2I. Sandra Laugier, «La vulnérabilité de l'ordinaire. Goffman lecteur d'Austin », dans Daniel Cefaï et Laurent Perreau (dir.), 20I2, p. 35I.

22. Tati, 1958, p. 3.

23. Tati, 1958 , p. II. 
Tati joint à l'infra-ordinaire, substrat social de son art, un désir de contrôle qui s'exprime à toutes les échelles de son travail, de l'architecture monumentale au pas de côté microscopique. Dans Playtime, l'improvisation caractéristique des passages et des rencontres urbaines se voit transformée en un ballet soigneusement chorégraphié, une partition interprétable plusieurs fois à l'identique.

Bausch travaillait également à partir d'un matériau vivant, cette fois extrait de son expérience de vie et de celles de ses danseurs. Voici comment elle décrivait sa démarche : «Ce que je fais : je regarde. Je n'ai fait que regarder les gens. Je n’ai fait que voir, ou essayer de voir les rapports humains, afin d'en parler ${ }^{24}$. » Chaque nouvelle création commençait par un long travail de recherche et d'expérimentation collective, à partir de questions qui, en 1978 pour Kontakthof, tournaient autour de la tendresse 25 . Proposant à ses acteurs-co-auteurs des images, des phrases, des thèmes, des mots-clefs, elle les invitait à plonger en eux et à en proposer des traductions par le mouvement. Elle observait et notait ces propositions, puis réalisait un travail conjoint de montage et de répétition. Chaque pièce s'est ainsi construite « non d'après un plan, mais par notre travail à tous. Et bien des choses dépendent de la composition de notre groupe, de ce que nous avons vécu ou de ce quelqu'un devait essayer de faire ${ }^{26}$. »

La genèse collective de chaque pièce passe dans la forme chorégraphique finale à travers les histoires des danseurs, leurs styles gestuels, leurs particularités corporelles, leur consistance existentielle, loin de la standardisation du corps du danseur classique. La traduction de Bausch opère néanmoins un figement du contingent en une écriture définitive qui, si elle porte en elle des traces du processus dont elle est née, en a transcendé les faiblesses, les fragilités. C’est cette discipline du corps que pointe le témoignage de Karlheinz Buchwald, du groupe de danseurs amateurs de plus de soixante-cinq ans avec qui la pièce écrite par la compagnie fut remontée en 2000 :

Si je me concentrais sur mon bras, c'était mon pied qui partait de travers. Si je pensais à sourire, je faisais de faux mouvements. Et apparemment, la liaison des synapses dans mon cerveau ne fonctionnait pas de manière à me faire mémoriser ces mouvements. Je prenais peu à peu conscience du haut degré d'entraînement, de concentration et de spécialisation des danseuses et des danseurs que nous avions vus et - à fort juste titre admirés sur scène ${ }^{27}$.

24. Raimund Hoghe, Pina Bausch. Histoires de théâtre dansé, Paris, L'Arche Éditeur, 1987, p. 8 .

25. Ibid., p. 22.

26. Ibid., p. 2I-22.

27. Jo Ann Endicott, « En scène, Mesdames et Messieurs », dans Kontakthof pour dames et messieurs de plus de 65 ans, Paris, L'Arche Éditeur, 2005, p. 95. 
Autrement que chez Tati, du fait de la performance vivante et charnelle de la danse, face à une pièce de Bausch, on retrouve ce sentiment paradoxal de vulnérabilité métaphysique, à la fois incarnée et annulée par la virtuosité du mouvement et de la mise en scène. Agamben synthétise ainsi la dualité présente dans la représentation visuelle du geste:

De fait, toute image est animée d'une polarité antinomique: elle est d'une part réification et annulation d'un geste (il s'agit alors de l'imago comme masque de cire mortuaire ou comme symbole), dont elle conserve d'autre part la dynamis intacte (ainsi dans les instantanés de Muybridge ou dans n'importe quelle photographie sportive) $)^{28}$.

Sur ce point, le médium photographique semble exacerber la logique de la représentation contre celle de la traduction. Chez Levé, l'objectification des corps fonctionne en effet à un degré bien plus élevé encore, la fixité de la photographie opérant chez lui « au carré ». Nous ne sommes pas face à un mouvement dont la vie serait domptée par sa répétition réglée. La performance elle-même est réduite à une pose dynamique, reproduisant précisément un dessin préalable de l'auteur. En conséquence, pour les photographies de la série « Rugby », l'effort pour les modèles ne consistait pas à « attraper une balle ou plaquer quelqu'un », mais à « simuler ces actes en arrêtant le cours du temps ${ }^{29} \gg$. La contrainte exercée sur le corps photographié est totale, et les détails vivants du visage, lieu de la subjectivité, disparaissent presque entièrement sous une impassibilité dépressive (voir les figures I3 et I4).

La dévitalisation du geste fait se matérialiser des mannequins, substituts humains dont les âmes se seraient absentées. Sur le plan plastique, les personnages de Levé sont des êtres tout à fait photographiques, présences presque sans profondeur, telles des poupées imprimées sur fond neutre, aux contours nets, à découper, à habiller et à «faire jouer » ensemble. Au niveau symbolique, ces mannequins mettent en valeur la fonction de représentation de soi du vêtement, ici presque entièrement réduit à une pure performance d'identité de classe ou de genre : costume-cravate pour les hommes de pouvoir, talons aiguilles pour les femmes érotisées... Levé pousse à l'extrême la logique de la transformation de situations sociales en objets abstraits et inertes. La précarité, la contingence et la fugacité de l'expérience sont annulées par l'autorité de la mise en scène en studio, dont toutes les composantes sont calculées et épurées : cadre, éclairage, disposition des corps, choix des couleurs...

28. Giorgio Agamben, « Notes sur le geste », $\operatorname{Trafic} \mathrm{n}^{\circ}$ I, hiver I99I, p. 34.

29. Levé, 2003 , p. 86. 

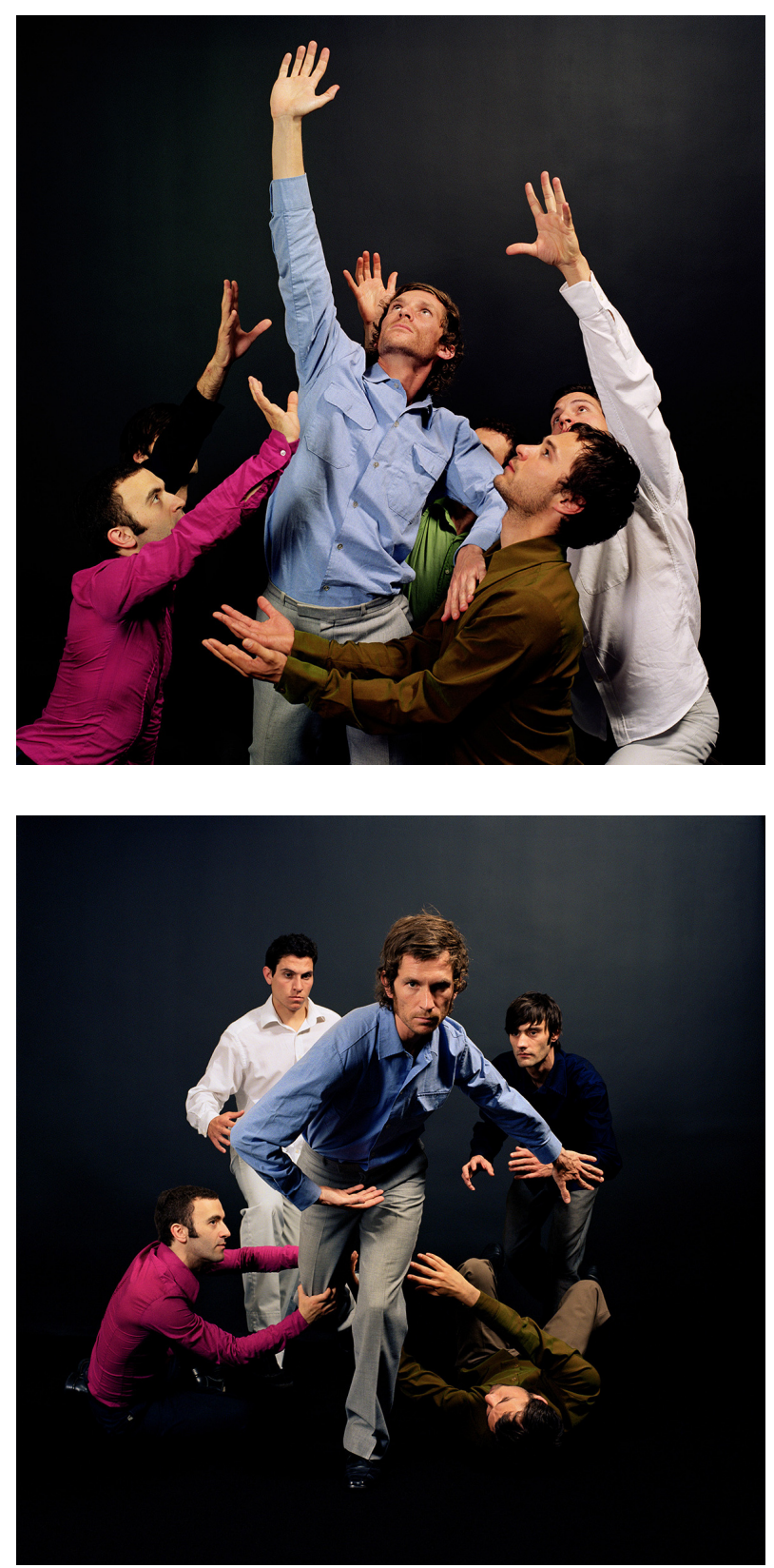

Fig. 13-I4. Édouard Levé, série Reconstitutions - Rugby, Sans titre, 2003, photographie couleur, IOo x IOO cm, édition à 5 exemplaires.

Reproduit avec l'aimable autorisation de la galerie Loevenbruck, Paris. 
À des degrés divers, les œuvres étudiées révèlent ainsi la violence du devenir chorégraphique des situations sociales: les chutes, les ratages, les manquements, les attitudes et les gestes improvisés par chacun au cours de l'interaction sociale sont ici simulés à travers le regard surplombant d'un auteur, et ce, à destination d'une classe sociale privilégiée, spectatrice de cinéma d'art, de danse contemporaine ou de photographie plasticienne. Devant Playtime, Kontakthof et Reconstitutions, les gestes sont devenus des images à voir, même chez Bausch, dont Brigitte Gauthier qualifie le travail de «théâtre d'images dansées ${ }^{30}$ ». Mais c'est que la traduction ne fonctionne pas sur le mode de la transposition analogique : l'imprévisible, l'inadéquation et l'écart qui fondent la dynamique vivante de l'interaction se déplacent. Le risque n'est plus ce qui guide les relations entre les corps sur scène. Il se glisse dans la situation sociale que constitue l'œuvre elle-même.

\section{DE LA DISTANCE SOCIALE À LA DISTANCE ESTHÉTIQUE}

Les œuvres étudiées ne se donnent pas à voir comme le résultat « transparent » d'un processus de traduction. Elles ont en commun de se mettre en scène en tant qu'expérience de traduction à laquelle le spectateur est incité à participer.

Les photographies d'Édouard Levé reprennent les codes d'imageries dominantes. Figures de pouvoir surplombantes, corps érotisés irréalistes et inaccessibles, ou peuples lointains dont les médias nous fournissent par intermittence quelques nouvelles : ces iconographies, plutôt que de donner à ceux qui les regardent le sentiment d'appartenir à un monde commun, fabriquent de la distance symbolique. Point de lien, de rencontre de part et d'autre de la surface de l'image, mais une asymétrie entre voyants ou voyeurs et visibles - asymétrie excitante dans le cas de la pornographie, angoissante ou violente dans le cas d'actualités politiques ou humanitaires. Levé, en reconstituant ces images comme autant de références génériques, tente d'en faire des objets de partage, des images relationnelles qui « regardent » les spectateurs, en même temps qu'elles ne se livrent jamais tout à fait à lui. Face aux Reconstitutions, le spectateur peut reconnaitre les codes culturels qui y sont à l'œuvre, et donc se sentir inclus dans l'élaboration d'un sens commun.

Au-delà de cette adresse directe, en partie ludique, l'alliance de la répétition du stéréotype contemporain et de l'étrangeté de sa stylisation crée une forme d'inquiétante étrangeté qui incite à s'engager dans une activité d'interprétation. Il n'est toutefois pas

30. Brigitte Gauthier, 2008, p. I2. 
question pour Levé de transmettre un message. Selon l'artiste, son travail ne constitue pas une « critique » ou une « dénonciation », mais une « anamorphose du sens » : « je cherche, par l'ambivalence sémiologique, à redevenir curieux à l'égard des images $^{31} \gg$. C'est donc le désir de regarder qu'il cherche à partager avec le spectateur à travers une pratique de l'«image pensive », qui est pour Jacques Rancière « une image qui recèle de la pensée non pensée, une pensée qui n'est pas assignable à l'intention de celui qui la produit et qui fait effet sur celui qui la voit sans qu'il la lie à un objet déterminé32 $\gg$.

D’une autre façon, on retrouve chez Tati ce désir d'impliquer le spectateur par l'exercice minutieux du regard. L'absence de procédés d'identification dans Playtime diminue la force de la «suspension temporaire de l'incroyance », qui règle l'immersion fictionnelle du cinéma classique. Plutôt qu'à se fondre dans le regard d'un personnage fortement impliqué dans l'action, le spectateur est invité à se projeter dans le regard de témoins, légèrement à distance. Pour reprendre la typologie goffmanienne, par l'intermédiaire de ces personnages récepteurs, le spectateur occupe la place d'un tiers autorisé, mais qui ne prend pas part directement à la situation sociale ${ }^{33}$, tel un passant qui observerait une situation dans l'espace public. Ce point de vue de spectateur-passant tient essentiellement, nous l'avons vu avec Michel Chion, au plan général, qui nous fait voir les corps à l'écran en pied, plutôt que de « nie[r] la distance entre nous et l'image ${ }^{34} \gg$, et donc entre nous et la situation sociale. Cette manière de (faire) voir prend forme de façon manifeste lors de plans récurrents dans Playtime où nous observons, depuis l'extérieur, des personnages évoluant à l'intérieur d'un bâtiment ou d'une pièce, souvent illuminée, derrière une vitre ou un surcadrage. Mise en scène cinématographique et mise en scène sociale révèlent explicitement leur devenir réciproque dans cette collusion du cadre lumineux de l'écran-vitrine et de la foule de spectateurs-passants. Lorsque Jean-Philippe Tessé écrit : « Notre monde est drôle, mais c'est une langue étrangère. Les films de Tati en sont la traduction ${ }^{35}{ } \gg$, il pointe le statut de Tati comme traducteur du quotidien, qui tel un marcheur curieux montrerait $\mathrm{du}$ doigt une situation pour attirer notre attention. Depuis cette position intermédiaire, à la fois inclus et en retrait, le spectateur de cinéma est mobilisé à travers

3I. Levé, 2003 , p. 86.

32. Jacques Rancière, Le spectateur émancipé, Paris, La fabrique éditions, 2008, p. II5.

33. Erving Goffman, Forms of Talk, Philadelphie, University of Pennsylvania Press, 198I, p. 226.

34. Chion, 1987, p. 5 I.

35. Tessé, 2007, p. 42. 
ses affects sociaux quotidiens : amusement, gêne, embarras, empathie, et ce, avec la légèreté permise par la fiction. Si Tati est un génie de la mise en scène cinématographique, la complicité qu'il cherche à instaurer avec les spectateurs par le rire repose d'abord sur la connaissance partagée des codes de la socialité ordinaire, c'està-dire sur le savoir de n'importe qui, plutôt que sur les références distinguées de l'art bourgeois.

Cette logique fait écho à la démarche de la nouvelle danse dont Bausch était la représentante et qui, selon les termes de Michèle Febvre, « [convoque] la banalité de nos comportements comme matériau chorégraphique et [installe] ainsi une proximité possible avec le spectateur ou un passage entre un corps sacré et un corps profane ${ }^{36} \gg$. Dans Kontakthof, le spectateur est même directement inclus dans la situation sociale enchâssée: à la fois bal où hommes et femmes se présentent les uns aux autres - comme l'évoquent le décor de salle de réception et les tenues de soirée - et spectacle où les danseurs se donnent en représentation pour le public. Chez Bausch, «Those who watch inhabit the same world as the characters $37 \gg:$ les acteurs se tournent en permanence vers l'assistance pour l'interpeller et exécuter pour elle des séquences dansées. Face à ces numéros successifs, les spectateurs sont investis d'un pouvoir de validation ou de remise en cause de l'image de soi ou de la « face ${ }^{8} \gg$, dont ils se saisissent alternativement avec bienveillance et violence en applaudissant ou en riant. Aux adresses directes et aux procédés comiques s'ajoutent également chez Bausch la mise en abyme du spectacle - projection cinématographique, spectacle de magie, concert - et la répétition de gestes ou de séquences chorégraphiques, qui participent à la mise à mal de l'illusion théâtrale classique (voir la figure $\mathbf{I}_{5}$ ).

Ces procédés de distanciation qui, chez Brecht, visaient à produire une prise de conscience intellectuelle des formes de l'aliénation, se mêlent chez Bausch à une force d'inspiration contraire, se situant du côté de l'incarnation, de la plasticité et du pur affect. Cette autre tendance, qui la rapproche du théâtre de la cruauté d'Artaud, cherche à réduire la distance entre les corps spectateurs et les corps dansants, pris ensemble dans une forme de communauté sensible et sensuelle. Dans Kontakthof, cela passe par des moments de pure jouissance et de lâcher prise physique, de mouvement compulsif, pulsionnel, mais aussi d'abattement ou d'épuisement, qui transmettent aux

36. Michèle Febvre, «Le corps dansant, aujourd'hui », Jeu : revue de théâtre, n 4I, 1986, p. $14-25$.

37. David W. Price, «The Politics of the Body: Pina Bausch's “Tanztheater” », Theatre Journal, vol. 42, n 3 , oct., I990, p. 326.

38. Erving Goffman, Les rites d'interaction, Paris, Les Éditions de Minuit, 1974, p. 9. 
corps du public une sorte d'énergie brute. Après une pièce de Bausch, les spectateurs parlent souvent du désir de se mettre à danser à leur tour, comme contaminés par un désir de mouvement. Dans Kontakthof, l'immersion des danseurs et des spectateurs dans une expérience commune passe aussi par l'usage que fait Bausch de la musique. Elle entretisse des chansons d'amour populaires, du tango et du swing des années i930, qui font appel à un imaginaire collectif mêlant amour et mélancolie.

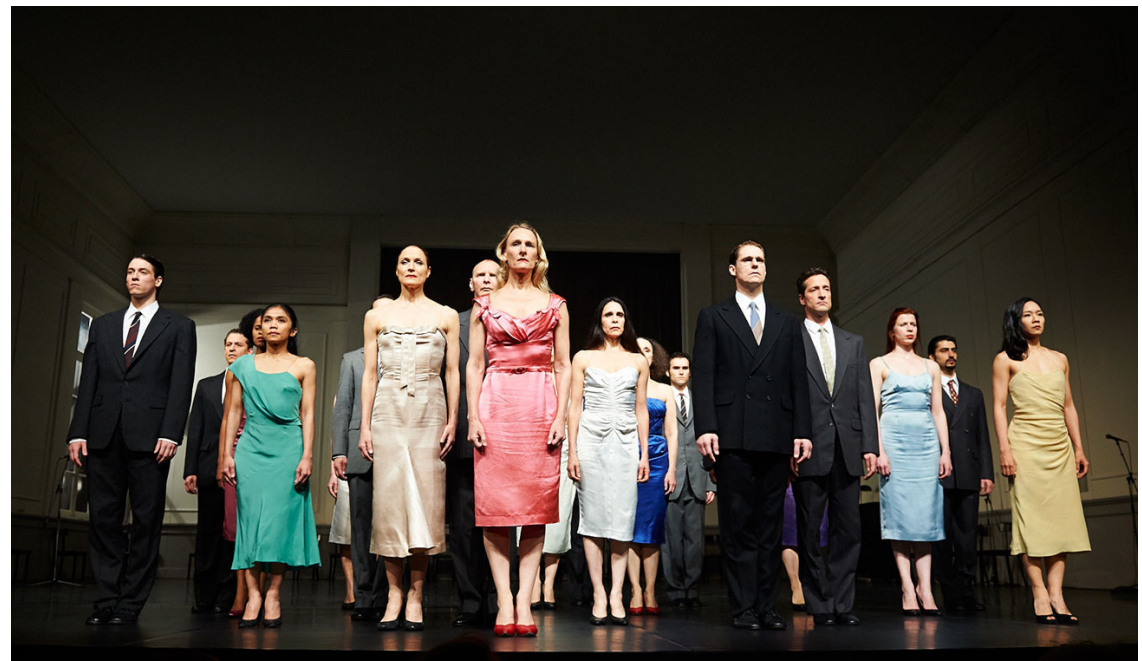

Fig. I5. Pina Bausch/Tanztheater Wuppertal, Kontakthof, 1978-2000-2008. Luminato Festival, Toronto, II-I4 juin 2014.

(c) David Leyes

Qu'ils soient comiques, réflexifs ou immersifs, ces différents procédés visent à inclure le regardeur, comme corps ou comme sujet, dans la relation sociale que constitue la traduction artistique. Ces gestes faits vers le spectateur constituent des tentatives de rapprochement ou de mise à distance, dont le contenu ne peut se construire que dans la rencontre indéterminée avec celui-ci. Pourtant, les œuvres de Tati et de Bausch ont souvent fait l'objet d'interprétations précises du point de vue référentiel. On a ainsi reproché au cinéaste de proposer une vision du monde conservatrice, anti-moderne. Certains commentateurs ont vu dans le travail de la chorégraphe une critique féministe de la domination patriarcale, tandis que d'autres lui ont au contraire reproché de reproduire sur scène une violence symbolique et physique sans la dénoncer clairement. Nombreux sont également ceux qui interprètent les œuvres de Bausch comme une représentation de la subjectivité allemande sinistrée d'après la Seconde Guerre mondiale. 
Mais, si Tati nous parle de la difficulté à nouer des liens dans l'anomie de la grande ville industrielle, on ne peut répondre de façon définitive à la question «Qu'en dit-il ? » Playtime propose plutôt au spectateur de faire l'expérience de ce monde moderne à travers une disposition sociale singulière, une attention amusée aux jeux des interactions humaines. Michel Chion voit ainsi les films de Tati comme de «purs entremetteurs ${ }^{39}$ », rappelant le souhait touchant du cinéaste : « Ce quej'aimerais, c'est que les gens se parlent en se montrant les choses $4^{\circ}$. » De ce travail d'observation partagée peut naître un comique ordinaire, qui fait que ceux qui rient et ceux dont on rit occupent des places interchangeables. C'est dans la construction performative de cette « démocratie comique ${ }^{4 \mathrm{I}} »$, selon la belle expression de Chion, que se joue le cœur de la dynamique de la traduction chez Tati. La structure égalitaire de son énonciation prend en compte ce qui « coince », ou « couine », dans la relation. D'une part, le brouillage de la communication verbale entre les personnages affecte également la compréhension du spectateur : les langues étrangères ne sont pas sous-titrées, et les voix se perdent dans le brouhaha de l'environnement. Mais, surtout, Tati prend en compte dans la relation qu'il propose la possibilité que les gags ne fassent pas rire :

Je comprends très bien pourquoi certains ont envie de partir à la moitié du film en disant : «Je ne comprends vraiment pas pourquoi les gens s'amusent », parce qu'il faut, sans doute, pour le goûter, apporter un peu d'observation de soi-même ${ }^{42}$.

S'il y a chez Tati une critique d'un certain monde industriel, c'est dans la conception de la traduction esthétique comme pratique du lien, partage d'un désir, à rebours de la communication par haut-parleurs de Tativille, qui ignore la distance et donc la réciprocité.

Bausch qualifiait quant à elle toute réduction de ses ouvres à un message unique de « dangereu[se] ${ }^{43}$ ». Son travail reposait sur le renouvellement permanent des questions — « Désespoir / C'est à moi / Tendre le pied / Faire des gratouillis / Nostalgie des lointains / Des poses effrontément décontractées... ${ }^{44} \gg-$ et la démultiplication des réponses dansées comme autant d'expériences possibles. D’où sa

39. Chion, 1987, p. 105.

40. Jacques Tati, « Le champ large. Entretien avec Jacques Tati par Jean-André Fieschi et Jean Narboni », Cabiers du Cinéma, n 199, mars 1968, cité dans Chion, 1987, p. 105.

4I. Chion, 1987, p. 28.

42. Tati, 1958 , p. 6.

43. Pina Bausch, dans Hoghe, 1987 , p. 18.

44. Ibid., p. 87 . 
réticence à traduire sa démarche en mots à l'occasion d'entretiens ou de présentations de ses spectacles : « Je ne peux quand même pas dire : voilà la route à suivre ${ }^{45}$ », disaitelle. De la même manière qu'entre les personnages la violence apparaît comme l'envers de la tendresse, ses spectateurs peuvent être tour à tour — ou singulièrement — séduits, révoltés ou dégoûtés par les images qu'elle propose. À propos de la répétition dans les pièces de Bausch, Cécile Léonardi souligne ainsi que ce procédé, qui consiste souvent à « mettre en scène des échanges qui ne vont nulle part, deux partenaires reprenant en boucle des gestes qui devraient logiquement faire évoluer leur rapport et qui, même réitérés, n'y changent rien ${ }^{46} \gg$, suscite une véritable consternation chez certains critiques. Ces réactions de rejet soulignent l'implication chez Pina Bausch du spectateur comme corps-sujet « regardé » par une œuvre, qui peut faire sienne l'adresse violente qu'elle met en scène. Comme si, par une sorte de contamination, l'impossible du rapport entre les corps dansants se traduisait par un empêchement dans la rencontre du spectateur avec la pièce.

Les traductions du social en esthétique que nous avons questionnées ne cherchent pas à calculer leurs effets sur le corps-sujet spectateur. Au contraire, elles réactivent, dans le rapport entre artistes-acteurs et spectateurs, le risque mis en scène dans les interactions entre les personnages. Dans le processus de traduction, les jeux de distance et de proximité, de tendresse et de violence, de séparation et de rencontre, qui sont le substrat de la socialité ordinaire, se déplacent dans l'espace de l'art pour donner vie à la relation entre le spectateur et l'œuvre. Autrement dit, la précarité et le potentiel de ratage de l'expérience sociale passent dans la dynamique de l'expérience esthétique elle-même : le geste projeté vers celui qui regarde prend en compte la possibilité du malaise, du rejet, de l'ennui, de l'incompréhension ou de l'indifférence.

En cela, l'adresse faite au spectateur par les artistes étudiés reprend la structure de l'adresse hétérolinguale, puisqu'elle se fait dans la distance et avec la possibilité de ne pas communiquer, et donc reconnaît l'altérité à soi-même et à l'autre ${ }^{47}$. Plus qu'en leur assignant des significations idéologiques particulières, c'est en mettant au jour la forme de cette adresse égalitaire qu'apparaît la dimension politique de telles œuvres. Ces pratiques artistiques de la traduction inventent performativement une communauté

45. Ibid., p. 2 I.

46. Cécile Léonardi, « De quelques figures de la répétition chez Pina Bausch », Repères, cabier de danse, janvier $2015, \mathrm{n}^{\circ} 35$, p. $2 \mathrm{I}$.

47. Jon Solomon, « Traduction, violence et intimité hétérolinguale », dans Rada Iveković(dir.), Translating Violence, Transversal, vol. II, $\mathrm{n}^{\circ} 7, \quad 2007$, www.eipcp.net/transversal/IIO7/solomon/fr (consultation le 25 avril 2016). 
politique d'auteurs-spectateurs, qui s'engagent ensemble dans la production permanente de contre-traductions du monde commun.

Certes, la traduction du social en esthétique dans ces ouvres contient une part de violence en tant qu'elles sont des remises en scène, par un auteur tout-puissant, de formes improvisées au quotidien par n'importe qui. Mais ce mouvement d'objectification donne naissance à une autre expérience de lien : l'objet artistique devient l'agent médiateur d'une relation vulnérable parce qu'indéterminée entre les auteurs, les interprètes et les spectateurs. Penser le geste artistique comme traduction plutôt que comme représentation permet donc d'insister sur la métamorphose d'une force en une autre, qui ne peut se réduire à la transposition mimétique d'une forme dans une autre. La traduction ne fait pas que mettre au jour le devenir chorégraphique des interactions sociales, elle fait également éclore la dynamique sociale de l'expérience esthétique. Les codes qui régissent les interactions en face à face sont traduits en codes esthétiques propres au cinéma, à la danse et à la photographie, qui contraignent en même temps qu'ils rendent possible la rencontre - plus ou moins conceptuelle, plus ou moins charnelle - avec un spectateur émancipé. La « démocratie comique » de Tati (Chion), l'adresse affectée chez Bausch, l'inquiétante étrangeté des clichés de Levé remettent en cause les rapports de pouvoir qui régissent la distribution des places sur la scène sociale ordinaire, en proposant de «concevoir une nouvelle scène de l'égalité où des performances hétérogènes se traduisent les unes dans les autres ${ }^{48} \gg$. Autrement dit, et pour filer la métaphore théâtrale évoquée en préambule à partir de l'œuvre de Lewis, plutôt que de faire passer les « extras » sur le devant de la scène, remettre en scène le quotidien, ce serait inventer un autre espace de jeu, à l'intérieur duquel personne n'est simple figurant.

48. Rancière, 2008, p. 28. 


\title{
L'art comme remise en scène de la vie quotidienne : traduire le social en esthétique
}

\author{
Camille Bui, Université Paris Diderot
}

\section{RÉSUMÉ}

Cet article explore, à travers le paradigme de la traduction, la mise en scène des interactions sociales dans Playtime de Jacques Tati (1967), Kontakthof de Pina Bausch (1978-2000-2008) et Reconstitutions d'Édouard Levé (1998-2003). Chacune de ces œuvres témoigne du désir de traduire le social en esthétique : il s'agit de dire l'ordinaire des jeux de distance et de proximité entre les corps, par le cinéma, la danse ou la photographie. Ce geste de traduction emprunte à la logique de la re-présentation qui réifie le social, mais il ne peut s'y réduire. Car la traduction vise aussi à reconduire, sur la scène de l'art, la dynamique du lien en tant qu'adresse faite à l'autre, au risque de son échec. Penser le geste artistique comme traduction permet d'être sensible au devenir chorégraphique des situations sociales, autant qu’à la socialité qui anime des œuvres qui nous émeuvent ou nous indifferent.

\section{ABSTRACT}

This article explores, through the paradigm of translation, the staging of social interactions in Playtime (Jacques Tati, 1967), Kontakthof (Pina Bausch, 1978-20002008), and Reconstitutions (Édouard Levé, 1998-2003). Each piece demonstrates the desire to translate the social into aesthetics: to tell with film, dance, or photography the ordinary games of distance and proximity between bodies. This translation gesture borrows from the logic of re-presentation that reifies the social, but cannot be reduced to it. Translation also aims to renew, in the experience of art, the dynamics of the social bond, that is, a move made towards the other, at the risk of its failure. To think art as translation renders us sensible to the choreographic qualities of social situations, as much as to the sociality of artworks that move us or leave us indifferent.

\section{NOTE BIOGRAPHIQUE}

Camille Bul est docteure en études cinématographiques et membre du CERILAC (Centre d'Études et de Recherches Interdisciplinaires en Lettres Arts Cinéma) de l'Université Paris Diderot. Elle est postdoctorante à l'Université de Montréal pour l'année 20ı6-20I7. Plusieurs de ses textes ont été publiés dans des revues telles que 
Annales de géographie («L'invention d'une rencontre entre le cinéma et la ville : la "symphonie urbaine" au tournant des années 1930 », 20I4), LISA (« Tongues Untied et Paris is Burning : les documentaires du New Queer Cinema », 20I4) ou Bioscope: South Asian Screen Studies ( U Umar Marvi and the Representation of Sindh: Cinema and Modernity in the Margins », 2014). Depuis décembre 2015, elle est également rédactrice pour Les Cabiers du cinéma. 